\title{
"Don't give me the theory, just tell me what to do in therapy!": The slippery slope challenge for the South African professions of Speech-Language Pathology and Audiology
}

The above frequent plea of the clinician attending a refresher course has resonated in my ears over the years. In this paper I address the reasons and frustrations caused by this enjoinder and in responding to the lead article, will reconsider priorities of our discipline and its implications for selection, training, funding and practice. I will argue that the discipline in this country is at an important crossroads and that future flourishing will depend on a proactive and firmly grounded commitment to scientific research.

Regardless of context, the twin disciplines of Speechlanguage Pathology and Audiology have always fought for scientific identity. The reasons for this have been multiple. The fact that their subject matter - human communication - is an interdisciplinary one has required a grounding in several main and historically secure fields: medicine, psychology, linguistics and physics to mention a few. Thus methods used for research in our disciplines have often been those borrowed from such fields and include a range of clinically based observations, experimental and descriptive designs. While there has been a historical effort to establish a unique identity for the disciplines, as reflected in some interesting debates on this topic, we are not yet there (Ringel, Trachtman, \& Prutting, 1984; Perkins, 1985; Siegel, 1987; Siegel \& Ingham, 1987).

The discipline in this country is 70 years old and this makes it a mere teenager in relation to some of its parents - and like a teenager, possibly still uncertain of its identity and autonomy and of which route to take towards this. If one looks at the history of our discipline, both here and elsewhere, we have followed and not taken the lead. Thus there has been a tendency to follow the current zeitgeist of medicine, psychology, and linguistics when searching for methods and explanatory frameworks. We take others' methods and theories and adapt them. In order to play the grant game and the publications game in research, this adherence to mainstream enables funding, acknowledgement, promotion and recognition. When you live at the cusp of another discipline you won't be noticed until you play that game and speak their language.

An example is offered from the field of aphasia, my own specialization, which can be characterized as having a number of distinct phases. Starting in the field of neurology with a strong tradition of case studies one can trace the influences of psychology, the preference for large scale group studies and the influences of early linguistic theory and the behavioural approach to communication of the 1960s. Pragmatics dominated the 1980s leading to current social approaches and we now see a return to the neural model with the advent of advanced functional neuroimaging. The influence of the WHO framework has been pervasive and is also mentioned in the lead article (see Penn, 2004a and Penn, 2005 for further consideration of these issues).

Any scholar of aphasia who is caught unawares and proposes a non-mainstream idea during a particular era has a challenging time getting an audience or arguing their case. Non mainstream ideas are tolerated or perhaps used as a platform for dialogue and debate. Those who work and think outside the box certainly add texture and depth to the discipline and serve (if a political analogy is allowed) as a type of opposition party in order to refresh and remind those who are heading towards a dictatorship. Often such streams of thought are published in different journals and at best attend parallel sessions at the same conferences. But such argument and debate we are told is essential for paradigm change and no discipline can thrive or grow without such mechanisms (Kuhn, 1970).

The search for a scientific and research identity and autonomy in our disciplines has been difficult, and remains in my opinion, elusive. The first reason may be because of the profoundly complex domain of study. Human behaviour is unpredictable and is influenced each day by a myriad of factors, as the lead article makes explicit. The influence of gender, race, poverty and socio political history have profound effects 
on human behaviour. To exclude these from research, to attempt to control for these variables or worse, to ignore them in explanations for what we find, leads to superficial and hegemonic conclusions. Secondly this makes the really important questions in our field (such as 'does therapy work?') almost impossible to answer to the satisfaction of the scientific community.

Take for example the heated debate around the (now) infamous Lancet study (Lincoln, Mulley, Jones, Mcguirk, Lendrem, \& Mitchell, 1984) which showed that therapy for aphasic patients in a certain setting did not work or at least did not work any better than no therapy. This controversial and (as it turns out) scientifically flawed study retrospectively had a major influence on the field. It caused sufficient levels of outrage and indignation among researchers and practitioners alike to generate a flurry of counter arguments and a body of well designed and reassuring studies on the efficacy of aphasia therapy (Wertz et al., 1986; Wertz, 1987). Thankfully, some people were reading the literature and justice was served. However the randomized controlled trial remains the gold standard for therapy efficacy in aphasia and hence most articles will be excluded from international data bases such as the Cochrane collaboration (Clarke \& Horton, 2001). As the lead article correctly suggests, the emphasis on evidence based practice (EBP) may have ignored some highly relevant material.

Further as the Lancet and other studies have shown, there has often been an insecurity in our disciplines which has come with the fact that we are a newer discipline and that we started off (and remain in some contexts) an "allied" discipline. Leary (1997) for example in relation to cerebral palsy describes the role of therapists in neurorehabilitation as "motivators, facilitators and informal counsellors", further suggesting that "it is the therapist's personality and approach to her patients rather than what she does which is to their advantage". Dim praise indeed, perpetuating an image of dependence, disempowerment and gender stereotype in the professions. But it is also possible that we have earned our own reputation and now we reap the fruits of our labour (or lack thereof). Unfortunately this appears particularly true for the discipline in our country. Several reasons suggest themselves.

Firstly there has been a reluctance to publish systematically. There are very few research-active individuals in the country with a sustained record of excellence. There are not enough postgraduate students in the field. This has been exacerbated by the introduction of community service and the Department of Health's insistence that clinicians may not register for a higher degree while fulfilling their community service (despite the profoundly valuable material they are working with). Outside the academic context, persons with higher degrees are not adequately recognized or remunerated. There is a considerable drop in the number of papers submitted to and published in this journal. There is not enough diversity in the papers submitted and it is not clear what the standard of evaluation have been applied. This trend is not unique to our context. There is a chronic shortage of doctoral students in the discipline in the United States (Gallagher, 2006). People would rather subscribe to clinical journals than to the Journal of Speech and Hearing Research.

Back home there are no research posts and little research funding available for our profession. University staffs, even those with particular research competence and leadership are obliged to focus on the clinical skills of their students. Researchers are leaving the field and joining other disciplines. It would be tempting to blame this state of affairs on what Gallagher (2006, p. 34) refers to as "the prevailing academic culture in the discipline that emphasizes clinical activities, clinical instruction and clinical career models" but this is not the whole story.

In the face of increasingly prescriptive curricula and training methods imposed by the professional bodies, some aspects of the curriculum have to give. Sadly some training departments are considering dropping the $4^{\text {th }}$ year research requirement in this country or limiting the project to a literature review or the development of a research proposal rather than the implementation of such a project- despite a crying need and opportunity for relevant research especially. This is not unique. Many training programmes in the United States have even dropped a research requirement at Masters level. The hardearned reputation of our profession may be losing ground.

Second, the adoption of methods from other disciplines appears to have been haphazard and often informed by necessity rather than systematic choice. There is as we all know a difference between clinical significance and research significance (Goldstein, 1990) but unless we can articulate this difference convincingly, our research will not be published. The problems we research require the application of novel research methodologies and it is probable that historical methods may have been too narrow. As we confront many of our current clinical challenges, methods and explanatory paradigms may extend beyond those disciplines which initially fed us and guided our direction. For example Earle (2001) has argued very convincingly for the value of teaching sociology to our disciplines as sociology is multi paradigmatic and inherently reflexive and is thus most suited to social behaviour. As I have argued elsewhere, (Penn, 2004b; Penn, 2005) there is a rich heritage of methods from other fields which can and should be brought to bear on some of the important issues especially in a culturally diverse context.

However most courses in research methods do not teach these (or do not teach them properly). Certainly the textbooks written for researchers in our field often specifically eschew or ignore these aspects. There may be a level of insecurity for the researcher who seeks valid applicable methods in the field of anthropology or interactional sociolinguistics, for example. And venturing into this new uncharted domain requires caution.

The choice of method should be driven by the research question. No researcher or discipline should abandon quantitative methods simply because they cannot understand them. I fear that the questioning of Mathematics as a potential entrance requirement at universities will have that impact, not just in our field but in other areas such as Education. The consequence of this is that researchers will choose elementary qualitative methods simply because they cannot choose anything else - 'if you don't (or can't) teach statistics then they will not do statistics'. As I see research emanating from different sources in this country, I believe that I can already see a direct relationship between the type of research conducted and the changes in admission criteria for the disciplines at training institutions.

There is a trend called "qualitative" in our field which does not really meet the rigorous requirements of proper research. It is really too easy to indicate that context is so important and our measures so complex that we don't need methods of the past. But there are equally many misconceptions about qualitative research which are rife. As many authors have pointed out there are a range of criteria for rigorous qualitative research (Johnson \& Long, 2000; Evans \& Pearson, 2001; Sharts-Hopko, 2002; Simmons Mackie \& Damico, 2003; Walsh \& Downe, 2006). Ethnography is not a "helicopter" visit (to use the words of a respected mentor, Peter Cleaton-Jones) but at least a six-month commitment to participant observation. 
Properly conducted scientific qualitative research is time-consuming and profoundly challenging and as I can personally attest, publishing it in our field is even more difficult! The guidelines that exist for evaluating the quality of qualitative research should be heeded and applied particularly by editors and reviewers of our journals if we are to make progress when judging the scientific merit of an article. The editors of this journal have an important role to play in quality control as well as in empowering the researcher. And in advancing research

Effective science is when you use the right method for the right question. The most difficult scientific choice occurs at the beginning of the project when you have to match the method to the question. Acknowledgements of the influence of cultural and contextual indices such as family, gender, artefact, history, geography, religion, education, myths and attitudes does not imply that these factors cannot be studied meaningfully in a quantitative way. It depends on the research question. I believe it is quite possible for a research question to have equipoise in terms of the method of choice. In other words it may well be answered in a number of different ways. If I ask whether a particular voice technique works for patients with Parkinson disease, I have a choice of design:

I could adopt a group approach and do it in a timeseries way (following a psychology tradition); I could use a control group who does not have the intervention (see Sapir, Spielman, Ramig, Story, \& Fox, 2007 for an example of this); I could measure outcomes on a spectrograph (drawing on technology and theory from linguistics); I could measure outcomes via open ended questionnaire and satisfaction scales (drawing on psychology and Quality of Life research); I could observe the use of voice in a number of contexts (using methods of description and observation); I could ask caregivers and family members of the participants about the social impact of the voice, or I could use any of the above in combination.

All of these would be acceptable methods provided there is a reason, and that reason may depend on the phase of the investigation, the scope of the study, the time frame and its clinical imperatives. What seems to matter is that I am aware that there are such options in research design and that there is scientific rigour as 1 move along. To ignore alternative designs, because of a limitation of knowledge is to deny the roots and the essence of our discipline. I believe it is not only acceptable, but a sign of academic maturity to adopt and combine methods and theory from some of our disciplinary partners. But when this is done, it must be done properly.

It seems a pity that the writers of the lead article do not explore more carefully the publications that have been produced in their own journal and other journals in the field, nor the issues which have been researched in this country. I think they would find some pioneering and honest attempts to resolve the challenges they have posed and to embrace and merge different paradigms.

Some common approaches to research deserve some attention. Therapy effectiveness studies have a long tradition and often take the format of single case studies (see Herson \& Barlow, 1984). Such studies are ideally suited for our field, because of the diversity of our clinic populations, the huge difficulty in finding sufficient participants for a group study and because of the compelling nature of therapy effectiveness. But there is often confusion between scientific single case studies and clinical case studies and as Finn, Bothe and Bramlett (2005) have pointed out, the field abounds with single case reports which are no more than a case description and which do not incorporate the essential elements of control which are the hallmarks of a true single case study including the multiple baseline measurement, randomization as well as clarity, reliability and validity of measure.

Another example comes from the measurement of the impact of training programmes (understandably a common interest in this context). A mistake is made to assume that a training programme is effective merely because there is a change in the participants' knowledge after that training programme. For example if there is a training programme to teach nurses about dysphagia and they show improved scores on a dysphagia quiz after that training, it proves nothing about the effectiveness of the training programme, only about the nurses' memory. Yet this is a standard design which I have seen published and would be classified as a weak or "pseudo" design (Finn et al., 2005). To turn this into a proper research study would involve comparing this programme with another, and measuring the impact on the quality of nursing care measured, either in terms of quantitative outcome (such as patient weight, or length of stay in hospital) or qualitative measures (such as patient satisfaction). The retention of such practice is also an important variable to measure.

Survey research is another method frequently used in our field. Knowledge, attitude and practice (KAP) research is frequently done, and its methods familiar and comforting but its relevance has to be questioned in some cases (Cleland, 1973). There seems to be little scientific merit in showing what we already know and what is instantly obvious. What do GPs know about language impairment? What do nurses know about dysphagia? (e.g. Pelletier, 2004) What services are lacking in rural areas? Do new graduates feel confident with hearing aid fittings? Unless you are the health minister (or perhaps in our current case, because you are the health minister) there is not much use exploring the attitudes of health professionals towards post shortages. Even armed with that outcome, there is little that can be done with that research to change practice or policy. We could generate a number of research questions which could be asked. But are all worth asking? Feasibility and relevance are different things and should not be confused.

This links closely the central theme of relevance discussed in the lead article. I agree that there is an urgency of service delivery in South Africa I am the first to agree we cannot ignore the political agenda of our context. Indeed statutory bodies and universities pick up such challenges and define and fund specific research thrusts. But these may not overlap with the ethical imperatives of our profession and some communities may hence be severely marginalized. One has to think for example of the impact of the AIDS epidemic on research funding opportunities outside that broad field in this country and the huge grant money in the US currently diverted to the field of cognitive neuroscience. Research goes where the money is and when last I checked, very little was flowing into research for the cerebral palsied in rural populations in this country despite a desperate need for understanding barriers to care (Barratt, 2007).

Sadly many practicing clinicians fail to see the relevance of research at all. This brings me back to the haunting recurrent words of the title of this article. Without the discipline of science, we will simply remain a group of 'practicing' clinicians and we will never achieve mastery or acknowledgement. We can 'practice' doing science but what is needed is for science to be done with authority, with an audience, with peer review, with argumentation and with energy.

\section{Conclusions}

This much I know. Unless our disciplines in this country strive 
for, sustain and improve their research agenda and profile, we are vulnerable in the extreme. We will be absorbed first into multidisciplinary schools and into multidisciplinary service cohorts and then eventually into technikons. Our functions will be (if they are not already) subsumed by ENTs, secretaries and assistants. The signs are there for all to see. We are expensive; we are probably too few in number to be heard. We have a limited proven effectiveness (Reilly, Douglas, \& Oates, 2004) and the leaders of our professional body appear to have completely different agendas from those of research excellence and scientific autonomy.

I end with some questions and challenges to the profession, the universities and the journal:

- Why is there such a shortage of postgraduate students?

- Why can't people do research without research funding?

- What steps have our national professional bodies taken to encourage, endorse and fund research?

- Why do people think that research is only for those who are not clinically inclined?

- What will happen when this ageing cohort of researchers in this country (amongst whom I proudly place myself) stops doing it?

- What will happen if the international profile and contribution that we have earned is eroded as we cease to publish in international journals and as our own journal readership dwindles?

As the lead article suggests, South Africa is a unique testing ground and clinician-researchers here have a huge potential to inform world practice and theory around issues of diversity, multilingualism and the unique disease profile. Based on our caseloads, our training, our daily interface and the splendid goals of our country's constitution, we could be world leaders in critical Speech-Language Pathology and Audiology (Penn, 2000).

Why is this not happening? While heavy caseloads are frequently cited as a reason why clinicians cannot engage in research, conversely that very fact should make the research process more compelling, accessible and easier. It is up to us to recognize and act on these opportunities and to publish them where they will make a difference.

Perkins (1985, p. 13) said that "Our field is haunted by a fallacy. We are predominantly a profession of practicing clinicians. That was not our origin. Beginning as scientists in search of understanding the nature of disordered speech were hardly out of our infancy before our scientific pursuits were outdistanced by the pressing need to help the millions who were speech and hearing handicapped".

Our identity is still a problem in this country. Review committees don't know where to place us. University restructuring committees and Faculty members are puzzled by our multidisciplinary curricula. Barring this journal we don't know where to publish our articles. There will be no progress in our identity for our professions until we have proven their scientific status. If we don't know what we are, we can hardly expect others to know. Being teenaged is not all bad but there is a critical time for introspection. Our identity has to have scientific credibility and should be respected so members of the profession can compete with others at scientific fora and for research money. There is a place for hard science. There is a place for other methods, but until it is done properly in either domain we will not get the recognition we need.
The Universities have a critical role to ensure research productivity in staff and to develop post graduate numbers. We need curriculum changes, additional funding and support for postgraduate study, representation on national research bodies and importantly, an attitude of curiosity. Above all there should be an appeal to explanatory theory, if necessary drawn from more mature, well grounded and respected scientific disciplines.

This journal has a critical role to play in encouraging and developing research which meets international standards. This research can (and should) be original and proactive and inform world practice about relevant issues of culture. The journal has a critical role to play in the profession in South Africa and in reminding its members about our origins. It has the responsibility not only of calling for papers but of setting the scientific tone of the profession, by advocating and endorsing the full range of scientific methods, for applying international standards of excellence in review of different methods and outcome research (Robey \& Schultz, 1998; Finn et al., 2005) and for asking for help when it is needed.

Our accountability to our clients and to our own profession's continued existence lies not solely in our clinical skills and our ability to convince the likes of Leary that we have nice personalities. I believe that some current developments in the South African profession are setting us back and I offer a challenge to the profession and its members and particularly to the journal to address and rectify those problems. Autonomy is the ability to thrive and "the capacity to think, decide and act on the basis of such thought and decision freely and independently" from the controlling interferences both by others and from personal limitations that prevent meaningful choice (after Gillon, 1985, p. 60). The autonomy of our profession lies primarily in demonstrating its scientific basis. It also involves establishing a strong relationship with its partners. To ignore this is to continue a slow descent into obscurity.

In conclusion I commend the authors of the lead article in posing a challenge. It is timely and should be heeded. I concur that it is time to tackle the challenges of context and to adopt frameworks of investigation and explanation which may be alternative to the mainstream positivistic ones. I do however recommend that this shift is done with caution and is grounded properly in the traditions of the disciplines framing such a shift. We cannot afford to be lukewarm about scientific endeavour.

The requirement of scientific rigour transcends any. one particular approach and remains the only way in which, our own disciplines can flourish through meaningful interface with other professions, with the international community and with the communities we serve.

\section{Acknowledgements}

Many of my ideas have emerged and slowly developed from an exciting opportunity I had to engage in a series of interactions on the topic "Is Speech Pathology a Science?" with Gerald Siegel, Carol Prutting and Gene Brutten. Their insights and ideas have profoundly affected our profession and my own career path and they taught and inspired research by example.

I am grateful to Jennifer Watermeyer for her research assistance and to Peter Fridjhon for his critical and helpful ear as well as many hours of shared enthusiasm on this topic.

Profound thanks to friend and colleague Dilys Jones who always acts as a sounding board for me and whom I consider to be a true example of the perfect clinician-researcher. 
Barratt, J. (2007). The experiences of caring for a child with cerebral palsy in Tonga, Mpumalanga. Unpublished Masters dissertation, University of the Witwatersrand.

Clarke, M., \& Horton, R. (2001). Bringing it all together: Lancet-Cochrane collaborate on systematic reviews. The Lancet, 357, 1728.

Cleland, J. (1973). A critique of KAP studies and some suggestions for their improvement. Studies in Family planning, 4, 42-47.

Earle, S. (2001). Teaching Sociology within the Speech and Language Therapy Curriculum. Education for Health, 14, 383-391.

Evans, D., \& Pearson, A. (2001). Systematic reviews of qualitative research. Clinical Effectiveness in Nursing, 5, 111-119.

Finn, P., Bothe, A., \& Bramlett, R. (2005). Science and Pseudoscience in Communication Disorders: Criteria and Applications. American Journal of Speech-Language Pathology, 14, 172-186.

Gallagher, T. (2006). US Doctoral Education: Critical Shortages and Plans for Reshaping the Future. Folia Phoniatrica et Logopaedia, 58, 32-35.

Gillon, R. (1985). Philosophical Medical Ethics. Chichester: Wiley.

Goldstein, H. (1990). Assessing clinical significance. In L.B. Olswang, C.K Thompson, S. F. Warren and N. J. Minghetti (Eds.), Treatment efficacy research in communication disorders (pp. 91-98). Rockville: ASHA.

Herson, J., \& Barlow, D. (1984). Single case experimental designs: Strategies for studying behaviour change ( $2^{\text {nd }}$ ed.). Elmsford: Pergamon Press.

Johnson, M., \& Long, T. (2000). Rigour, reliability and validity in qualitative research. Clinical Effectiveness in Nursing, 4, 30-37.

Kuhn, T. (1970). The Structure of Scientific Revolutions $\left(2^{\text {nd }}\right.$ ed.). Chicago: University of Chicago Press.

Leary, P. (1997). Interventions for children with neurodevelopmental delay South African Medical Journal, 87, 1680-1683.

Lincoln, N., Mulley, G., Jones, A., Mcguirk, E., Lendrem, W., \& Mitchell, J. (1984). Effectiveness of speech therapy for aphasic stroke patients: A randomised controlled trial. The Lancet, 323, 1197-1200.

Pelletier, C. (2004). What do certified nurse assistants actually know about dysphagia and feeding home residents? American Journal of SpeechLanguage Pathology, 13, 99-113.

Penn, C, (2000). Cultural narratives: bridging the gap. South African Journal of Communication Disorders: Special edition on Communication Disorders in Multilingual Populations, 47, 71-78.
Penn, C. (2004a). Context, culture and conversation. In S. Byng and J. Duchan (Eds.), Challenging aphasia therapies: Broadening the discourse and extending the boundaries (pp. 83-100). Psychology Press: London

Penn, C. (2004b). 'Festina lente': a case for making haste slowly in reflective practice. A response to Ferguson and Armstrong. International Journal of Language and Communication Disorders, 30, 490-497.

Penn, C. (2005). Who's tired of the WHO?: A commentary on Ross and Wertz, "Advancing appraisal: Aphasia and the WHO." Aphasiology, 19, 875-879.

Perkins, W. (1985). From clinical dispenser to clinical scientist. Seminars in Speech and Language, 6, 13-21.

Reilly, S., Douglas, J., \& Oates, J. (2004). Evidence Based Practice in Speech Pathology. London: Whurr

Ringel, R., Trachtman, L., \& Prutting, C. (1984). The Science in Human Communication Sciences. ASHA, December, 33-37.

Robey, R., \& Schultz, M. (1998). A model for conducting clinical outcome research: An adaptation of the standard protocol for use in aphasiology. Aphasiology, 12, 787-810.

Sapir, S., Spielman, J., Ramig, L., Story, B., \& Fox, C. (2007). Effects of intensive voice treatment (the Lee Silverman Voice Treatment [LSVT]) on vowel articulation in dysarthric individuals with idiopathic Parkinson disease: Acoustic and perceptual findings. Journal of Speech and Hearing Research, 50, 89-912.

Sharts-Hopko, N. (2002). Assessing Rigor in Qualitative Research. Journal of the Association of Nurses in Aids Care, 13, 84-86.

Siegel, G. (1987). The limits of science in communication disorders. Journal of Speech and Hearing Disorders, 52, 306-312.

Siegel, G., \& Ingham, R. (1987). Theory and science in communication disorders. Journal of Speech and Hearing Disorders, 52, 99-104.

Simmons-Mackie, N., \& Damico, J. (2003). Contributions of qualitative research to the knowledge base of normal communication. American Journal of Speech - Language Pathology, 12, 144-154.

Walsh, D., \& Downe, S. (2006). Appraising the quality of qualitative research. Midwifery, 22, 108-119.

Wertz, R. (1987). Language treatment for aphasia is efficacious, but for whom? Topics in Language Disorders, 8, 1-10.

Wertz, R., Weiss, D., Aten, J., Brookshire, R., Garcia-Bunuel, L., Holland, A., et al. (1986). Comparison of clinic, home, and deferred language treatment for aphasia: Veterans Administration cooperative study. Archives of Neurology, $43,653-658$. 